Washington University School of Medicine Digital Commons@Becker

\title{
Stenting versus aggressive medical therapy for intracranial arterial stenosis
}

Colin P. Derdeyn

Washington University School of Medicine in St. Louis

et al

Follow this and additional works at: https://digitalcommons.wustl.edu/open_access_pubs Please let us know how this document benefits you.

\section{Recommended Citation}

Derdeyn, Colin P. and et al, "Stenting versus aggressive medical therapy for intracranial arterial stenosis." The New England Journal of Medicine. 365, 11. 993-1003. (2011).

https://digitalcommons.wustl.edu/open_access_pubs/2958

This Open Access Publication is brought to you for free and open access by Digital Commons@Becker. It has been accepted for inclusion in Open Access Publications by an authorized administrator of Digital Commons@Becker. For more information, please contact vanam@wustl.edu. 
ORIGINAL ARTICLE

\section{Stenting versus Aggressive Medical Therapy for Intracranial Arterial Stenosis}

\author{
Marc I. Chimowitz, M.B., Ch.B., Michael J. Lynn, M.S., Colin P. Derdeyn, M.D., \\ Tanya N. Turan, M.D., David Fiorella, M.D., Ph.D., Bethany F. Lane, R.N., \\ L. Scott Janis, Ph.D., Helmi L. Lutsep, M.D., Stanley L. Barnwell, M.D., Ph.D., \\ Michael F. Waters, M.D., Ph.D., Brian L. Hoh, M.D., J. Maurice Hourihane, M.D., \\ Elad I. Levy, M.D., Andrei V. Alexandrov, M.D., Mark R. Harrigan, M.D., \\ David Chiu, M.D., Richard P. Klucznik, M.D., Joni M. Clark, M.D., \\ Cameron G. McDougall, M.D., Mark D. Johnson, M.D., G. Lee Pride, Jr., M.D., \\ Michel T. Torbey, M.D., M.P.H., Osama O. Zaidat, M.D., \\ Zoran Rumboldt, M.D., and Harry J. Cloft, M.D., Ph.D., \\ for the SAMMPRIS Trial Investigators*
}

A BSTRACT

\section{BACKGROUND}

Atherosclerotic intracranial arterial stenosis is an important cause of stroke that is increasingly being treated with percutaneous transluminal angioplasty and stenting (PTAS) to prevent recurrent stroke. However, PTAS has not been compared with medical management in a randomized trial.

\section{METHODS}

We randomly assigned patients who had a recent transient ischemic attack or stroke attributed to stenosis of 70 to $99 \%$ of the diameter of a major intracranial artery to aggressive medical management alone or aggressive medical management plus PTAS with the use of the Wingspan stent system. The primary end point was stroke or death within 30 days after enrollment or after a revascularization procedure for the qualifying lesion during the follow-up period or stroke in the territory of the qualifying artery beyond 30 days.

\section{RESULTS}

Enrollment was stopped after 451 patients underwent randomization, because the 30 -day rate of stroke or death was $14.7 \%$ in the PTAS group (nonfatal stroke, $12.5 \%$; fatal stroke, $2.2 \%$ ) and 5.8\% in the medical-management group (nonfatal stroke, 5.3\%; non-stroke-related death, $0.4 \%)(\mathrm{P}=0.002)$. Beyond 30 days, stroke in the same territory occurred in 13 patients in each group. Currently, the mean duration of followup, which is ongoing, is 11.9 months. The probability of the occurrence of a primary end-point event over time differed significantly between the two treatment groups $(\mathrm{P}=0.009)$, with 1-year rates of the primary end point of $20.0 \%$ in the PTAS group and $12.2 \%$ in the medical-management group.

\section{CONCLUSIONS}

In patients with intracranial arterial stenosis, aggressive medical management was superior to PTAS with the use of the Wingspan stent system, both because the risk of early stroke after PTAS was high and because the risk of stroke with aggressive medical therapy alone was lower than expected. (Funded by the National Institute of Neurological Disorders and Stroke and others; SAMMPRIS ClinicalTrials.gov number, NCT00576693.)
The authors' affiliations are listed in the Appendix. Address reprint requests to $\mathrm{Dr}$. Chimowitz at the Medical University of South Carolina Stroke Program, 19 Hagood Ave., Harborview Office Tower, Suite 501, Charleston, SC 29425, or at mchimow@musc.edu.

\section{*The investigators in the Stenting and Aggressive Medical Management for Pre- venting Recurrent Stroke in Intracranial Stenosis (SAMMPRIS) trial are listed in the Supplementary Appendix, available at NEJM.org.}

This article (10.1056/NEJMoal105335) was published on September 7, 2011, and updated on July 5, 2012, at NEJM.org.

N Engl J Med 2011;365:993-1003

Copyright $\odot 2011$ Massachusetts Medical Society. 
THEROSCLEROTIC INTRACRANIAL ARterial stenosis is one of the most common causes of stroke worldwide ${ }^{1-6}$ and is associated with a high risk of recurrent stroke. ${ }^{7-9}$ Patients with a recent transient ischemic attack (TIA) or stroke and severe stenosis (70 to $99 \%$ of the diameter of a major intracranial artery) are at particularly high risk for recurrent stroke in the territory of the stenotic artery (approximately 23\% at 1 year) despite treatment with aspirin and standard management of vascular risk factors. ${ }^{8,10}$ Therefore, alternative therapies are urgently needed for these patients.

Two strategies have emerged for the treatment of high-risk patients: aggressive medical therapy (combination antiplatelet therapy and intensive management of risk factors) and percutaneous transluminal angioplasty and stenting (PTAS). Over the past decade, intracranial PTAS has increasingly been used in clinical practice in the United States and other countries. ${ }^{11-19}$ Currently, the self-expanding Wingspan stent (Boston Scientific) is the only device approved by the Food and Drug Administration (FDA) for use in patients with atherosclerotic intracranial arterial stenosis; it has been available since 2005 for the treatment of patients with 50 to $99 \%$ stenosis who have had a TIA or stroke while receiving antithrombotic therapy. ${ }^{20}$

Because of uncertainty regarding the safety and efficacy of aggressive medical management alone as compared with aggressive medical management plus PTAS with the use of the Wingspan stent system, we began a randomized trial in November 2008 to compare these two treatments in highrisk patients with intracranial arterial stenosis. On April 5, 2011, the trial's independent data and safety monitoring board recommended that enrollment be stopped because of safety concerns regarding the risk of periprocedural stroke or death in the PTAS group and because futility analyses indicated that there was virtually no chance that a benefit from PTAS would be shown by the end of the follow-up period if enrollment continued. Although follow-up of patients is ongoing, the clinical importance of these findings mandated the reporting of the current results.

\section{METHODS}

STUDY DESIGN AND OVERSIGHT

Details of the trial design have been published previously. ${ }^{21}$ This study is an investigator-initiated, randomized, clinical trial funded by the National Institute of Neurological Disorders and Stroke and conducted at 50 sites in the United States. Stryker Neurovascular (formerly Boston Scientific Neurovascular) provided the study devices and supplemental funding for third-party distribution of devices and continues to provide funding for site monitoring and auditing of the study. The Investigator-Sponsored Study Program of AstraZeneca donates rosuvastatin (Crestor) to study patients. Other industry partners are listed at the end of the article. None of the industry partners participated in the design of the trial or in the analysis or reporting of the results. The study protocol, available with the full text of this article at NEJM.org, was approved by the institutional review board at each site, and the FDA issued an investigational device exemption to enable us to carry out the study.

The data and safety monitoring board met every 6 months and reviewed monthly reports to monitor the study's progress and the accumulated data. Two interim efficacy analyses were planned when approximately $33 \%$ and $66 \%$ of the required primary end points had occurred. There were no prespecified stopping rules for safety.

\section{STUDY PATIENTS}

Eligible patients had a TIA or nondisabling stroke within 30 days before enrollment, attributed to angiographically verified stenosis of 70 to $99 \%$ of the diameter of a major intracranial artery. The other eligibility criteria are provided in the study protocol. All the patients gave written informed consent to participate, and patients who did not undergo diagnostic angiography as part of routine care gave consent for angiography as part of the study protocol.

\section{TREATMENTS}

\section{Aggressive Medical Management}

The rationale for the medical-management regimen and details on the management of risk factors in the study patients have been published previously. ${ }^{21-23}$ Medical management is identical in the two groups and consists of aspirin, at a dose of $325 \mathrm{mg}$ per day; clopidogrel, at a dose of $75 \mathrm{mg}$ per day for 90 days after enrollment; management of the primary risk factors (elevated systolic blood pressure and elevated low-density lipoprotein [LDL] cholesterol levels); and management of secondary risk factors (diabetes, elevated non-high-density lipoprotein [non-HDL] cholesterol levels, smoking, excess weight, and insufficient exercise) with the 
help of a lifestyle modification program. With respect to the primary risk factors, we targeted a systolic blood pressure of less than $140 \mathrm{~mm} \mathrm{Hg}$ ( $<130 \mathrm{~mm} \mathrm{Hg}$ in the case of patients with diabetes) and an LDL cholesterol level of less than $70 \mathrm{mg}$ per deciliter (1.81 mmol per liter). We provide the aspirin, clopidogrel, one drug from each major class of antihypertensive agents, rosuvastatin, and the lifestyle program to the study patients.

\section{PTAS Procedure}

PTAS was performed by neurointerventionists who were selected by a committee of experienced neurointerventionists on the basis of their review of procedure notes and outcomes for the 20 most recent consecutive cases of intracranial stenting or angioplasty (if angioplasty had been performed to treat atherosclerosis) performed by the neurointerventionists under consideration. Further details regarding the credentialing process and the monitoring of the interventionists' performance of PTAS during the trial have been published previously. ${ }^{21}$ Patients who were randomly assigned to PTAS were required to undergo the procedure within 3 business days after randomization. Patients who were not taking clopidogrel at a dose of $75 \mathrm{mg}$ each day for at least 5 days before PTAS were given a 600-mg loading dose of clopidogrel between 6 and 24 hours before PTAS. Details of the procedure, which was performed under general anesthesia with the use of the Gateway PTA Balloon Catheter and Wingspan Stent System (both manufactured by Boston Scientific Corporation), and of the care of the patients after the procedure are provided in the protocol.

\section{FOLLOW-UP AND ASSESSMENT OF OUTCOME}

Patients were evaluated at the time of study entry, at 4 days, and at 30 days and have continued to be evaluated every 4 months; patients undergo assessments until 90 days after a primary end point occurs, the patient dies, 3 years of follow-up have been completed, or the close-out visit for the trial is held, which will occur when the last patient enrolled has been followed for 1 year. At followup visits, patients are examined by study neurologists who also manage the patients' vascular risk factors. If a stroke is suspected during the follow-up period, the patient is examined by the study neurologist, and magnetic resonance imaging (MRI) or computed tomography (CT) of the brain is typically performed. Because the treatment assignment is known to the study neurologist, we require that a second site neurologist, who is not aware of the treatment assignments, evaluate any patient who has had a prolonged TIA (lasting more than 1 hour) or mild ischemic stroke (an increase in the patient's score on the National Institutes of Health Stroke Scale [NIHSS] of $<4$ points from the score at study entry), since these events may be difficult to classify. (The NIHSS is a 42-point scale that quantifies neurologic deficits in 11 categories, with higher scores indicating more severe deficits.) The assessments of both neurologists are sent for central adjudication.

All the end points are adjudicated by independent panels of neurologists and cardiologists who are not informed of the treatment assignments. The primary end point is stroke or death within 30 days after enrollment or after a revascularization procedure for the qualifying lesion during the follow-up period (i.e., angioplasty for symptomatic restenosis in a patient in the PTAS group or placement of a stent in a patient in the medicalmanagement group) or ischemic stroke in the territory of the qualifying artery between day 31 and the end of the follow-up period. Ischemic stroke is defined as a new focal neurologic deficit of sudden onset, lasting at least 24 hours, that is not associated with a hemorrhage on CT or MRI of the brain. Ischemic strokes are further classified by the neurologic adjudicators as being either in the territory or out of the territory of the qualifying artery. Symptomatic brain hemorrhage is defined as a parenchymal, subarachnoid, or intraventricular hemorrhage detected on CT or MRI that is associated with a seizure or with new neurologic signs or symptoms lasting at least 24 hours; it is included as a primary end point only if it occurs within 30 days after enrollment or within 30 days after a revascularization procedure for the qualifying lesion during the follow-up period.

\section{STATISTICAL ANALYSIS}

The mean length of follow-up was designed to be 2 years. In the Warfarin-Aspirin Symptomatic Intracranial Disease trial (WASID; ClinicalTrials.gov number, NCT00004728), ${ }^{7}$ the rate of the same primary end point among patients with symptoms within 30 days before enrollment and 70 to $99 \%$ stenosis was $29 \%$ at 2 years. With adjustment of that rate to account for an estimated 15\% relative reduction in risk with aggressive medical management, the projected rate of the primary end point in the medical-management group was $24.7 \%$ at 2 years. We estimated that we would need to en- 
roll 382 patients in each group for the study to have $80 \%$ power to show a relative reduction of $35 \%$ with PTAS in the risk of the primary end point, assuming a $5 \%$ crossover rate from the medical-management group to the PTAS group and a $2 \%$ loss to follow-up, with the use of a twosided log-rank test, at a type I error rate of 0.05 .

We tested the primary hypothesis by comparing the rate of the primary end point between the two treatment groups using a two-sided log-rank test. Data from patients who were lost to followup or who withdrew consent were censored at the last contact date. Secondary end points were analyzed with the use of the same techniques. The probability of a primary end point by 30 days after enrollment was compared between the two treatment groups with the use of a $z$ test. All analyses were performed in the intention-to-treat population unless otherwise specified. All reported $P$ values are two-sided and have not been adjusted for multiple testing.

\section{RESULTS}

\section{PATIENTS}

Of the 451 patients who underwent randomization, 227 were assigned to the medical-management group and 224 to the PTAS group (see the figure in the Supplementary Appendix, available at NEJM.org). There were no significant differences between the two groups with respect to any of the baseline characteristics of the patients (Table 1), but the groups did differ significantly at various times during the trial with respect to some of the measures of risk factors (Table 2, and expanded version of Table 2 in the Supplementary Appendix). Of the 224 patients in the PTAS group, 16 (7.1\%) did not have a stent placed (the procedure was not performed in 4 patients, the procedure was aborted before the lesion was accessed in 7 , and angioplasty alone was performed in 5). Of the 227 patients in the medical-management group, $9(4.0 \%)$ underwent PTAS after a TIA during the follow-up period.

\section{END POINTS}

The data on end points presented below are based on all adverse events as of April 28, 2011, when the last patient enrolled completed the 30-day evaluation. Data from patients without events who had follow-up visits after that date were censored as of April 28, 2011, for all analyses.
Primary End Points within 30 Days after Enrollment The probability of the primary end point was $14.7 \%$ (involving 33 patients) in the PTAS group and 5.8\% (involving 13 patients) in the medical-management group ( $\mathrm{P}=0.002)$ (Fig. 1 and Table 3). There were five stroke-related deaths in the PTAS group (2.2\%) and one non-stroke-related death in the medicalmanagement group $(0.4 \%)$. A total of 10 of the 33 strokes in the PTAS group (30.3\%) and none of the 12 in the medical-management group were symptomatic brain hemorrhages $(\mathrm{P}=0.04$ by Fisher's exact test). Further details of the types of strokes that occurred in the two groups are provided in Table 3.

Of the 33 strokes in the PTAS group that occurred within 30 days after enrollment, 25 occurred within 1 day after the procedure and 8 occurred 2 to 6 days later. The 33 strokes occurred at 25 investigational sites. Of 6 sites at which more than 1 periprocedural stroke occurred, 5 were among the highest-enrolling sites. The 30-day rate of stroke among patients who underwent PTAS was $13.5 \%$ at the highest-enrolling sites (i.e., at 12 sites that enrolled half the patients) and $14.7 \%$ at the other sites (38 sites that enrolled the other half) $(\mathrm{P}=0.77)$. The risk of periprocedural stroke did not diminish over the course of the enrollment period ( $\mathrm{P}=0.20$ by the Cochran-Armitage test for trend).

\section{Primary End Points beyond 30 Days}

Beyond 30 days, nonfatal ischemic strokes in the territory of the qualifying artery have occurred in 13 patients in each group (Table 3). The probability of the occurrence of a primary end point over the entire follow-up period after enrollment differed significantly between the two treatment groups $(\mathrm{P}=0.009)$, with 1-year rates of the primary end point of $20.0 \%$ in the PTAS group and $12.2 \%$ in the medical-management group (Fig. 1 and Table 3). An as-treated analysis that excluded 11 patients in the PTAS group who did not undergo angioplasty or have a stent placed (3 of whom had a stroke) and 9 patients in the medical-management group who underwent PTAS during the follow-up period ( 3 of whom had a stroke after PTAS) showed the same result $(\mathrm{P}=0.009)$.

\section{Secondary End Points and Other Adverse Events}

Table 3 shows the secondary end points and other major adverse events during the follow-up period in each group. The rates of any stroke and of any 


\begin{tabular}{|c|c|c|}
\hline Characteristic & $\begin{array}{c}\text { Medical-Management } \\
\text { Group } \\
(\mathrm{N}=227)\end{array}$ & $\begin{array}{l}\text { PTAS Group } \\
(\mathrm{N}=224)\end{array}$ \\
\hline Age $-y r$ & $59.5 \pm 11.8$ & $61.0 \pm 10.7$ \\
\hline Male sex — no. (\%) & $145(63.9)$ & $127(56.7)$ \\
\hline \multicolumn{3}{|l|}{ Race - no. (\%)† } \\
\hline Black & $50(22.0)$ & $55(24.6)$ \\
\hline White & $161(70.9)$ & $160(71.4)$ \\
\hline Other & $16(7.0)$ & $9(4.0)$ \\
\hline Hypertension — no. (\%) & $203(89.4)$ & $201(89.7)$ \\
\hline Diabetes — no. (\%) & $103(45.4)$ & $106(47.3)$ \\
\hline Lipid disorder — no. (\%) & $203(89.4)$ & $194(86.6)$ \\
\hline \multicolumn{3}{|l|}{ Smoking history — no./total no. (\%) } \\
\hline Never & $78 / 227(34.4)$ & $90 / 223(40.4)$ \\
\hline Former & $80 / 227(35.2)$ & $79 / 223(35.4)$ \\
\hline Current & $69 / 227(30.4)$ & $54 / 223(24.2)$ \\
\hline History of coronary artery disease - no. (\%) & $59(26.0)$ & $47(21.0)$ \\
\hline History of stroke other than qualifying event — no. (\%) & $58(25.6)$ & $60(26.8)$ \\
\hline \multicolumn{3}{|l|}{ Qualifying event - no. (\%) } \\
\hline Stroke & $152(67.0)$ & $142(63.4)$ \\
\hline TIA & $75(33.0)$ & $82(36.6)$ \\
\hline $\begin{array}{l}\text { Already receiving antithrombotic therapy at time of qualifying event - } \\
\text { no. (\%) }\end{array}$ & $141(62.1)$ & $145(64.7)$ \\
\hline \multicolumn{3}{|l|}{ Time from qualifying event to randomization - days } \\
\hline Median & 7 & 7 \\
\hline Interquartile range & $4-19$ & $4-16$ \\
\hline \multicolumn{3}{|l|}{ Symptomatic qualifying artery — no. (\%) } \\
\hline Internal carotid & $49(21.6)$ & $45(20.1)$ \\
\hline Middle cerebral & $105(46.3)$ & $92(41.1)$ \\
\hline Vertebral & $22(9.7)$ & $38(17.0)$ \\
\hline Basilar & $51(22.5)$ & 49 (21.9) \\
\hline \multicolumn{3}{|l|}{ Stenosis of symptomatic qualifying artery: } \\
\hline Mean percentage stenosis & $81 \pm 7$ & $80 \pm 7$ \\
\hline \multicolumn{3}{|l|}{ Distribution — no./total no. (\%) } \\
\hline 70-79\% stenosis & $102 / 227(44.9)$ & $107 / 223(48.0)$ \\
\hline $80-89 \%$ stenosis & $97 / 227$ (42.7) & $92 / 223(41.3)$ \\
\hline $90-99 \%$ stenosis & $28 / 227$ (12.3) & $24 / 223(10.8)$ \\
\hline
\end{tabular}

* Plus-minus values are means \pm SD. Baseline characteristics of the two groups were compared with the use of either an independent groups t-test (for means) or a chi-square test (for percentages). None of the characteristics differed significantly between the groups ( $P>0.05$ for all comparisons). PTAS denotes percutaneous transluminal angioplasty and stenting.

$\dagger$ Race was self-reported.

$\mp$ Stenosis was quantified on the basis of a reading of the angiogram by the site interventionist. 


\begin{tabular}{|c|c|c|c|c|}
\hline \multirow[t]{2}{*}{ Variable } & \multicolumn{2}{|c|}{$\begin{array}{c}\text { Medical-Management } \\
\text { Group } \\
(\mathrm{N}=227)\end{array}$} & \multicolumn{2}{|c|}{$\begin{array}{l}\text { PTAS Group } \\
(\mathrm{N}=\mathbf{2 2 4})\end{array}$} \\
\hline & Baseline & $4 \mathrm{Mo}$ & Baseline & $4 \mathrm{Mo}$ \\
\hline \multicolumn{5}{|l|}{ Clinical factor } \\
\hline \multicolumn{5}{|l|}{ Blood pressure } \\
\hline No. of patients evaluated & 227 & 179 & 220 & 173 \\
\hline Systolic - mm Hg & $146.8 \pm 21.8$ & $134.8 \pm 17.0$ & $143.9 \pm 20.6$ & $133.1 \pm 15.9$ \\
\hline Diastolic — mm Hg† & $82.3 \pm 12.0$ & $77.3 \pm 10.0$ & $77.9 \pm 10.7$ & $76.2 \pm 9.7$ \\
\hline \multicolumn{5}{|l|}{ Lipidsł } \\
\hline No. of patients evaluated & 226 & 175 & 219 & 174 \\
\hline LDL cholesterol - mg/dl & $97.7 \pm 36.6$ & $72.8 \pm 26.0$ & $96.3 \pm 38.5$ & $75.9 \pm 40.9$ \\
\hline HDL cholesterol — mg/dl & $38.8 \pm 10.1$ & $41.9 \pm 11.4$ & $37.8 \pm 10.6$ & $43.2 \pm 13.3$ \\
\hline Non-HDL cholesterol - mg/dl & $116.6 \pm 40.3$ & $90.9 \pm 30.8$ & $116.6 \pm 43.9$ & $94.3 \pm 50.2$ \\
\hline \multicolumn{5}{|c|}{ Glycated hemoglobin in patients with diabetes $\mathbb{3}$} \\
\hline No. of patients evaluated & 98 & 47 & 102 & 50 \\
\hline Level of glycated hemoglobin — \% & $8.3 \pm 2.3$ & $7.5 \pm 2.0$ & $7.9 \pm 2.1$ & $7.8 \pm 2.4$ \\
\hline \multicolumn{5}{|l|}{ Body-mass index } \\
\hline No. of patients evaluated & 227 & 180 & 224 & 170 \\
\hline Value & $30.7 \pm 6.3$ & $30.4 \pm 6.4$ & $30.3 \pm 6.2$ & $30.0 \pm 6.2$ \\
\hline \multicolumn{5}{|l|}{ Lifestyle factor } \\
\hline No. of patients evaluated & 227 & 181 & 223 & 173 \\
\hline Current smoker — \% & 30.4 & 20.4 & 24.2 & 17.3 \\
\hline Moderate or vigorous exercise — \%\| & 29.1 & 56.6 & 34.2 & 56.1 \\
\hline
\end{tabular}

* Plus-minus values are means \pm SD. Risk-factor measures in both groups at 30 days and at 1 year are provided in Table 2 in the Supplementary Appendix. To convert the values for cholesterol to millimoles per liter, multiply by 0.02586 . PTAS denotes percutaneous transluminal angioplasty and stenting.

$\dagger$ The difference in diastolic blood pressure at baseline between the two groups is significant $(P<0.001)$.

$\checkmark$ Lipid levels at baseline and 4 months were measured at the Central Lipid Laboratory, with low-density lipoprotein (LDL) cholesterol levels measured directly. Non-high-density lipoprotein (non-HDL) cholesterol is total cholesterol minus HDL cholesterol, or the sum of LDL cholesterol and very-low-density lipoprotein cholesterol.

$\int$ Levels shown are baseline and 6-month levels, rather than baseline and 4-month levels (the protocol did not require levels to be measured at 4 months). Diabetes was defined according to the 2010 criteria of the American Diabetes Association.

9 The body-mass index is the weight in kilograms divided by the square of the height in meters.

|| Moderate or vigorous exercise was defined as a score on the Physician-based Assessment and Counseling for Exercise (PACE) evaluation of 4 to 8 , with 4 indicating vigorous exercise less than three times per week or moderate exercise less than five times per week and 8 indicating vigorous exercise at least 3 days a week for at least the previous 6 months. Examples of moderate exercise include brisk walking, gardening, and slow cycling for at least 10 minutes; examples of vigorous exercise include jogging, running, and fast cycling for at least 20 minutes. A total of 182 patients were included in the medical-management group at 4 months.

major hemorrhage were significantly higher in the PTAS group than in the medical-management group. The difference between the two groups in the rate of death or any stroke (16.3\% vs. $23.2 \%)$ was not significant $(\mathrm{P}=0.06)$.

\section{DISCUSSION}

Contrary to what we hypothesized, the results of this trial showed that aggressive medical therapy was superior to PTAS with the use of the Wing- 
span system in high-risk patients with intracranial stenosis, because the rate of periprocedural stroke after PTAS was higher than expected and the rate of stroke in the medical-management group was lower than estimated. The 30-day rate of stroke or death in the PTAS group (14.7\%) is substantially higher than the rates previously reported with the use of the Wingspan stent in the phase I trial and in two registries (rates ranging from $4.4 \%$ to $9.6 \%$ ). ${ }^{10,20,25}$ The higher rate in the current study does not reflect inexperience of the operators, because most of the interventionists who participated in the registries also participated in this trial, and all the interventionists in this trial were credentialed to participate on the basis of evidence of their experience. In addition, the rates of periprocedural stroke did not decline over the course of the enrollment period and did not differ significantly between high-enrolling sites and low-enrolling sites in this trial.

One possible explanation for the higher rate of periprocedural stroke in this trial as compared with the registries is that all the patients in this study had stenosis of 70 to $99 \%$ and recent symptoms, whereas the registries included patients with stenosis of 50 to $99 \%$ and symptoms that had occurred more than 30 days before enrollment. Recent symptoms may be a marker for unstable plaque, which could increase the risk of distal embolism during stenting, as has been reported with extracranial carotid stenting. ${ }^{26,27}$ Another explanation for the higher rate of periprocedural stroke in this trial is that the rigorous protocol for evaluating events (i.e., evaluation of all potential end points by neurologists, the adjudication process, and sitemonitoring visits) could have resulted in the detection of some milder strokes that may not have been detected in the registries. However, the percentage of primary end-point strokes in the PTAS group that were disabling or fatal $(35 \%$; 16 of 46 patients) is higher than the percentage of primary end-point strokes that were categorized as major in the stenting group (21\%) or the endarterectomy group $(28 \%)$ in a recent randomized trial involving patients with extracranial carotid stenosis. ${ }^{28}$

The rate of stroke in the medical-management group was much lower than expected. Patients in the WASID trial with the same entry criteria who were treated with aspirin or warfarin and standard management of risk factors had a 30-day rate of stroke or death of $10.7 \%$ and a 1-year rate of the

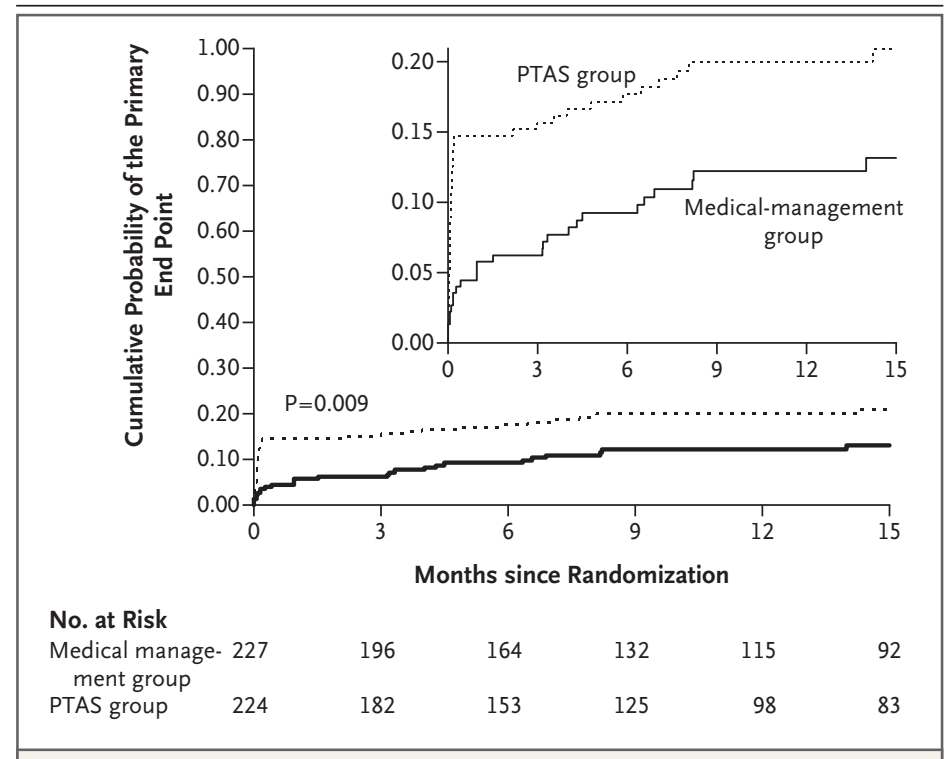

Figure 1. Kaplan-Meier Curves for the Cumulative Probability of the Primary End Point, According to Treatment Assignment.

The primary end point was stroke or death within 30 days after enrollment or after a revascularization procedure for the qualifying lesion during the follow-up period or stroke in the territory of the qualifying artery beyond 30 days. The curves were truncated at 15 months because relatively few patients have been followed beyond this time and there have only been two primary end-point events beyond 15 months, both in the group receiving percutaneous transluminal angioplasty and stenting (PTAS) (one at 26.1 months and one at 26.2 months). The maximum duration of follow-up is 28.9 months for the group receiving medical management only and 28.1 months for the PTAS group. The inset shows the same data on an enlarged segment of the $y$ axis.

primary end point of $25 \% .{ }^{10}$ In contrast, the corresponding rates in the medical-management group in this trial were $5.8 \%$ and $12.2 \%$. Although we expected the rate of stroke to be reduced with intensive management of risk factors - on the basis of post hoc analyses from the WASID trial that suggested that lowering LDL cholesterol and systolic blood pressure could reduce the risk of stroke ${ }^{22,29}$ — we were surprised at the extent and rapidity of the reduction. It is also possible that the combination of aspirin and clopidogrel played an important role in lowering the early risk of stroke. This is supported by the results of a study of transcranial Doppler ultrasonography involving patients with recently symptomatic intracranial stenosis, which showed that aspirin and clopidogrel, as compared with aspirin alone, reduced the frequency of ipsilateral distal microemboli. ${ }^{30}$ The effect of the lifestyle modification program on the outcome can be determined only at the end 


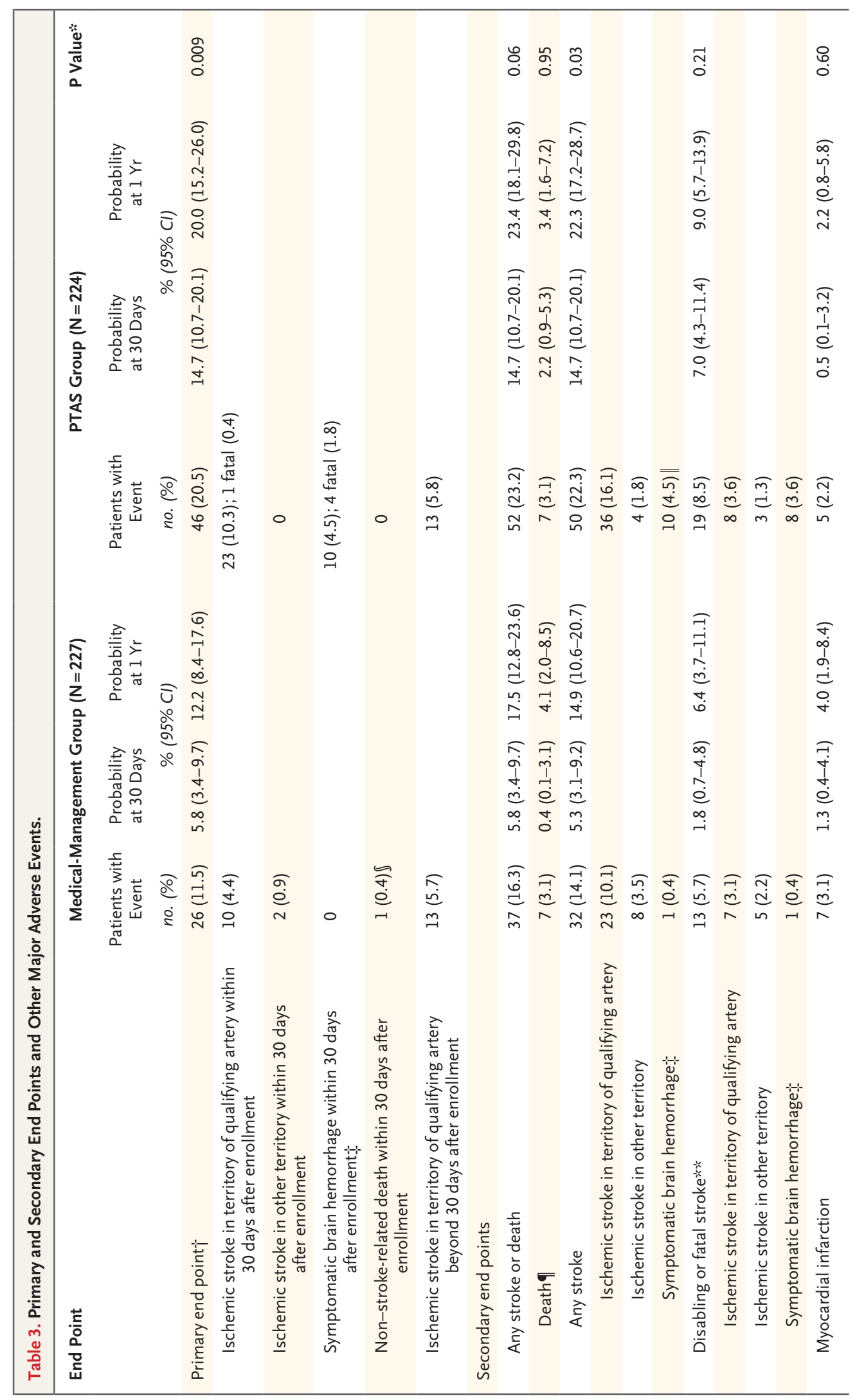




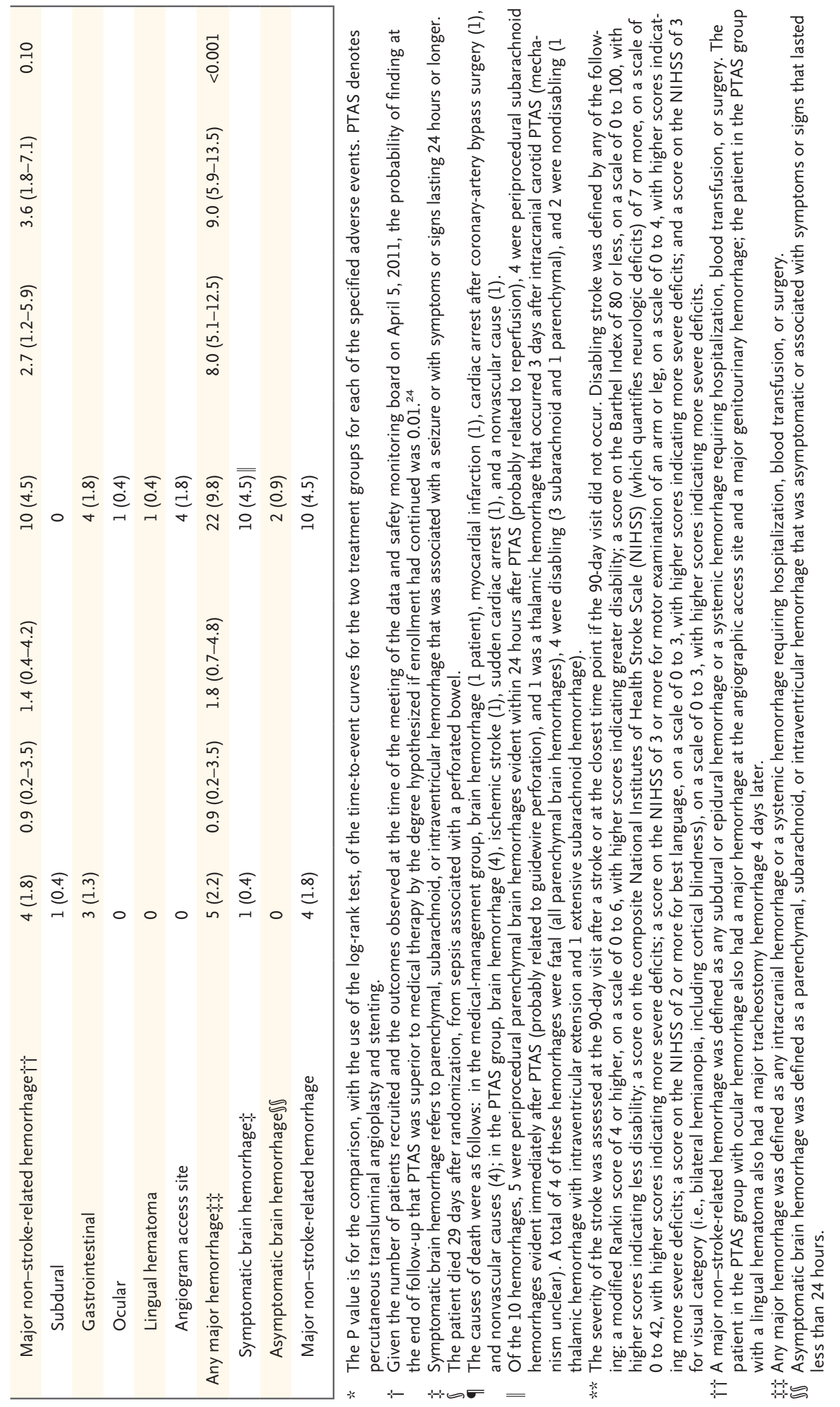

N ENGLJ MED 365;11 NEJM.ORG SEPTEMBER 15, 2011 
of the follow-up period, but it is unlikely that it contributed to a reduction in the risk of stroke in the medical-management group within 30 days after enrollment.

The difference between the treatment groups in the rate of the primary end point is driven by the early events, since the rates of the primary end point beyond 30 days are currently similar in the two groups. However, fewer than half the patients have been followed for longer than 1 year. Therefore, continued follow-up of the patients who are currently enrolled will be important to determine the long-term outcome in the two groups. Among patients who are receiving medical management only, progression of stenosis may occur over time that could result in a stroke from a distal embolism or hypoperfusion. ${ }^{31-35}$ Among patients in whom a stent has been placed, restenosis occurs in 25 to $30 \%$ within 6 months after intracranial PTAS $^{36,37}$ and could also lead to later stroke.

Patients with symptoms that occurred more than 30 days before enrollment or with stenosis of 50 to $69 \%$ of an intracranial artery were excluded from this trial because their risk of stroke while receiving standard medical care is relatively low (approximately 3 to $9 \%$ at 1 year ${ }^{8,21}$ ), making it unlikely that they would benefit from PTAS. These patients could have an even lower risk of stroke if they received aggressive medical therapy. This trial did not evaluate angioplasty alone or other devices (e.g., balloon-mounted stents) that are used off-label to treat patients with intracranial stenosis. Although these devices may have benefits over the Wingspan system (e.g., singlestep delivery and deployment of the stent and less residual stenosis after the procedure), none have been compared with medical management.

The current results of this trial indicate that medical therapy as delivered in this trial is superior to PTAS with the Wingspan stent system, which is associated with a high risk of periprocedural stroke or death in this population. Although not all the components of the aggressive medical regimen used in this trial may be easy to duplicate in clinical practice, essential elements can readily be adopted, including adding clopidogrel to aspirin for the first 90 days and following the trial's protocol with respect to lowering blood pressure and LDL cholesterol in order to achieve target levels that are based on national guidelines. ${ }^{38,39}$

Supported by a research grant (U01 NS058728) from the National Institute of Neurological Disorders and Stroke (NINDS). In addition, the following institutions received Clinical and Translational Science Awards, funded by the National Institutes of Health, that provided local support for the evaluation of patients in the trial: Medical University of South Carolina (UL1RR029882), University of Florida (UL1RR029889), University of Cincinnati (UL1RR029890), and University of California, San Francisco (UL1RR024131). This research is also supported by the Investigator-Sponsored Study Program of AstraZeneca, which donates rosuvastatin (Crestor) to study patients. Stryker Neurovascular (formerly Boston Scientific Neurovascular) provided study devices and supplemental funding for third-party distribution of devices and continues to provide funding for site monitoring and study auditing, and Nationwide Better Health-INTERVENT provides the lifestyle modification program to the study at a discounted rate. The Regulatory and Clinical Research Institute (Minneapolis) provided assistance in designing the site monitoring processes and performs the site monitoring visits. The Cooperative Studies Program Clinical Research Pharmacy Coordinating Center of the Department of Veterans Affairs (Albuquerque, NM) handles the procurement, labeling, distribution, and inventory management of the study devices and rosuvastatin. Walgreens pharmacies provide study medications, except rosuvastatin, to patients at a discounted price (paid for by the study). The Physician-based Assessment and Counseling for Exercise (PACE) self-assessment forms for physical activity and smoking cessation were provided by the San Diego Center for Health Interventions.

Disclosure forms provided by the authors are available with the full text of this article at NEJM.org.

We thank the patients for participating in this study; Drs. Oscar Benavente, Carole White, Robert Hart, Pablo Pergola, and Ana Roldan of the Secondary Prevention of Small Subcortical Strokes trial (SPS3, NCT00059306) for assisting with the development and implementation of the SAMMPRIS blood-pressure management protocol; Drs. George Howard and Thomas Brott for providing advice on study design and other issues on the basis of their experience with the Carotid Revascularization Endarterectomy versus Stenting Trial (CREST, NCT00004732); and Rie Calcaterra for helping with the SAMMPRIS grant application and with launching the trial.

\section{APPENDIX}

The authors' affiliations are as follows: the Departments of Neurosciences (M.I.C., T.N.T.) and Radiology (Z.R.), Medical University of South Carolina, Charleston; the Department of Biostatistics and Bioinformatics, Emory University Rollins School of Public Health, Atlanta (M.J.L., B.F.L.); Mallinckrodt Institute of Radiology and the Departments of Neurology and Neurosurgery, Washington University School of Medicine, St. Louis (C.P.D.); the Department of Neurosurgery, State University of New York, Stony Brook (D.F.); the National Institute of Neurological Disorders and Stroke, National Institutes of Health, Bethesda, MD (L.S.J.); the Department of Neurology (H.L.L.) and the Department of Neurological Surgery and the Dotter Interventional Institute (S.L.B.), Oregon Health Sciences University, Portland; the Departments of Neurology and Neuroscience (M.F.W.) and Neurosurgery (B.L.H.), University of Florida, Gainesville; Dent Neurological Institute (J.M.H.) and the Department of Neurosurgery, University of Buffalo (E.I.L.) — both in Buffalo, NY; the Departments of Neurology (A.V.A.) and Neurosurgery (M.R.H.), University of Alabama, Birmingham; the Departments of Neurology (D.C.) and Radiology (R.P.K.), Methodist Hospital, Houston; the Departments of Neurology (J.M.C.) and Neurosurgery (C.G.M.), Barrow Neurological Institute, Phoenix, AZ; the Departments of Neurology (M.D.J.), Neurotherapeutics (M.D.J.), Radiology (G.L.P.), and Neurosurgery (G.L.P.), University of Texas Southwestern Medical Center, Dallas; the Departments of Neurology (M.T.T., O.O.Z.), Radiology (O.O.Z.), and Neurosurgery (O.O.Z.), Medical College of Wisconsin, Milwaukee; and the Department of Radiology, Mayo Clinic, Rochester, MN (H.J.C.). 
1. Wong LK. Global burden of intracranial atherosclerosis. Int J Stroke 2006;1: 158-9.

2. Gorelick PB, Wong KS, Bae HJ, Pandey DK. Large artery intracranial occlusive disease: a large worldwide burden but a relatively neglected frontier. Stroke 2008 ; 39:2396-9.

3. Arenillas JF. Intracranial atherosclerosis: current concepts. Stroke 2011;42 Suppl:S20-S23.

4. Kim JS, Kang DW, Kwon SU. Intracranial atherosclerosis: incidence, diagnosis and treatment. J Clin Neurol 2005;1:1-7.

5. Sacco RL, Kargman D, Gu Q, Zamanillo MC. Race-ethnicity and determinants of intracranial atherosclerotic cerebral infarction: the Northern Manhattan Stroke Study. Stroke 1995;26:14-20.

6. Meseguer E, Lavallée PC, Mazighi M, et al. Yield of systematic transcranial Doppler in patients with transient ischemic attack. Ann Neurol 2010;68:9-17.

7. Chimowitz MI, Lynn MJ, HowlettSmith H, et al. Comparison of warfarin and aspirin for symptomatic intracranial arterial stenosis. N Engl J Med 2005;352: 1305-16.

8. Kasner SE, Chimowitz MI, Lynn MJ, et al. Predictors of ischemic stroke in the territory of a symptomatic intracranial arterial stenosis. Circulation 2006;113:55563.

9. Wong KS, Li H. Long-term mortality and recurrent stroke risk among Chinese stroke patients with predominant intracranial atherosclerosis. Stroke 2003;34 2361-6.

10. Zaidat OO, Klucznik R, Alexander MJ, et al. The NIH registry on use of the Wingspan stent for symptomatic $70-99 \%$ intracranial arterial stenosis. Neurology 2008;70:1518-24.

11. Rasmussen PA, Perl J II, Barr JD, et al. Stent-assisted angioplasty of intracranial vertebrobasilar atherosclerosis: an initial experience. J Neurosurg 2000;92:771-8.

12. Levy EI, Hanel RA, Boulos AS, et al. Comparison of periprocedure complications resulting from direct stent placement compared with those due to conventional and staged stent placement in the basilar artery. J Neurosurg 2003;99:65360.

13. Fiorella D, Chow MM, Anderson M, Woo H, Rasmussen PA, Masaryk TJ. A 7-year experience with balloon-mounted coronary stents for the treatment of symptomatic vertebrobasilar intracranial atheromatous disease. Neurosurgery 2007; 61:236-42.

14. SSYLVIA Study Investigators. Stenting of Symptomatic Atherosclerotic Lesions in the Vertebral or Intracranial Arteries (SSYLVIA): study results. Stroke 2004;35: 1388-92.
15. Kim JK, Ahn JY, Lee BH, et al. Elective stenting for symptomatic middle cerebral artery stenosis presenting as transient ischaemic deficits or stroke attacks: short term arteriographical and clinical outcome. J Neurol Neurosurg Psychiatry 2004;75:84751.

16. Suh DC, Kim JK, Choi JW, et al. Intracranial stenting of severe symptomatic intracranial stenosis: results of 100 consecutive patients. AJNR Am J Neuroradiol 2008;29:781-5.

17. de Rochemont RM, Turowski B, Buchkremer M, Sitzer M, Zanella FE, Berkefeld J. Recurrent symptomatic high-grade intracranial stenosis: safety and efficacy of undersized stents - initial experience. Radiology 2004;231:45-9.

18. Jiang WJ, Xu XT, Du B, et al. Comparison of elective stenting of severe vs moderate intracranial atherosclerotic stenosis. Neurology 2007;68:420-6.

19. Yu SC, Leung TW, Lee KT, Hui JW, Wong LK. Angioplasty and stenting of atherosclerotic middle cerebral arteries with Wingspan: evaluation of clinical outcome, restenosis, and procedure outcome. AJNR Am J Neuroradiol 2011;32:753-8.

20. Bose A, Hartmann M, Henkes $H$, et al. A novel, self-expanding, nitinol stent in medically refractory intracranial atherosclerotic stenoses: the Wingspan study. Stroke 2007;38:1531-7.

21. Chimowitz MI, Lynn MJ, Turan TN, et al. Design of the stenting and aggressive medical management for preventing recurrent stroke in intracranial stenosis trial. J Stroke Cerebrovasc Dis 2011;20:357-68.

22. Chaturvedi S, Turan TN, Lynn MJ, et al. Risk factor status and subsequent vascular events in patients with symptomatic intracranial stenosis. Neurology 2007;69: 2063-8.

23. Gordon NF, Salmon RD, Franklin BA, et al. Effectiveness of therapeutic lifestyle changes in patients with hypertension, hyperlipidemia, and/or hyperglycemia. Am J Cardiol 2004;94:1558-61.

24. Lan KKG, Wittes J. The B-value: a tool for monitoring data. Biometrics 1988;44: 579-85.

25. Fiorella D, Levy EI, Turk AS, et al. US multicenter experience with the Wingspan stent system for the treatment of intracranial atheromatous disease: periprocedural results. Stroke 2007;38:881-7. 26. Gray WA, Yadav JS, Verta P, et al. The CAPTURE registry: predictors of outcomes in carotid artery stenting with embolic protection for high surgical risk patients in the early post-approval setting. Catheter Cardiovasc Interv 2007;70:1025-33.

27. Topakian R, Strasak AM, Sonnberger $\mathrm{M}$, et al. Timing of stenting of symptomatic carotid stenosis is predictive of 30 day outcome. Eur J Neurol 2007;14:672-8.
28. Brott TG, Hobson RW II, Howard G, et al. Stenting versus endarterectomy for treatment of carotid-artery stenosis. N Engl J Med 2010;363:11-23. [Errata, N Engl J Med 2010;363:198, 498.]

29. Turan TN, Cotsonis G, Lynn MJ, Chaturvedi S, Chimowitz M. Relationship between blood pressure and stroke recurrence in patients with intracranial arterial stenosis. Circulation 2007;115:2969-75.

30. Wong KS, Chen C, Fu J, et al. Clopidogrel plus aspirin versus aspirin alone for reducing embolisation in patients with acute symptomatic cerebral or carotid artery stenosis (CLAIR study): a randomised, open-label, blinded-endpoint trial. Lancet Neurol 2010;9:489-97.

31. Derdeyn CP. Mechanisms of ischemic stroke secondary to large artery atherosclerotic disease. Neuroimaging Clin N Am 2007;17:303-11.

32. Wang X, Lam WW, Fan YH, Graham CA, Rainer TH, Wong KS. Topographic patterns of small subcortical infarcts associated with MCA stenosis: a diffusionweighted MRI study. J Neuroimaging 2006; 16:266-71.

33. Choi JW, Kim JK, Choi BS, et al. Angiographic pattern of symptomatic severe M1 stenosis: comparison with presenting symptoms, infarct patterns, perfusion status, and outcome after recanalization. Cerebrovasc Dis 2010;29:297-303.

34. Lee DK, Kim JS, Kwon SU, Yoo SH, Kang DW. Lesion patterns and stroke mechanism in atherosclerotic middle cerebral artery disease: early diffusion-weighted imaging study. Stroke 2005;36:2583-8.

35. Famakin BM, Chimowitz MI, Lynn MJ, Stern BJ, George MG. Causes and severity of ischemic stroke in patients with symptomatic intracranial arterial stenosis. Stroke 2009;40:1999-2003.

36. Turk AS, Levy EI, Albuquerque FC, et al. Influence of patient age and stenosis location on Wingspan in-stent restenosis. AJNR Am J Neuroradiol 2008;29:23-7.

37. Levy EI, Turk AS, Albuquerque FC, et al. Wingspan in-stent restenosis and thrombosis: incidence, clinical presentation, and management. Neurosurgery 2007;61:64450.

38. Chobanian AV, Bakris GL, Black HR, et al. The Seventh Report of the Joint National Committee on Prevention, Detection, Evaluation, and Treatment of High Blood Pressure: the JNC 7 report. JAMA 2003;289:2560-72. [Erratum, JAMA 2003; 290:197.]

39. Grundy SM, Cleeman JI, Merz CNB, et al. Implications of recent clinical trials for the National Cholesterol Education Program Adult Treatment Panel III guidelines. Circulation 2004;110:227-39. [Erratum, Circulation 2004;110:763.]

Copyright (c) 2011 Massachusetts Medical Society. 